\title{
Les Olympiades internationales de physique
}

Moins célèbres que celles des jeux sportifs, les Olympiades Internationales de Physique (IPhO) existent néanmoins depuis 1967 et fédèrent désormais près de quatre-vingt-dix délégations nationales. Les IPhO 2016 sont organisées par la Suisse et le Liechtenstein, et se dérouleront du 10 au 18 juillet à Zurich. Une délégation française y sera bien sûr présente, comme c'est le cas depuis 2004, année où la France a renoué avec les IPhO sous l'impulsion de Sophie Rémy.

\section{Les épreuves et récompenses}

Les équipes comportent au plus cinq candidats. La compétition, individuelle, consiste en deux épreuves de cinq heures : une théorique et une expérimentale, qui sont rédigées par le pays hôte. Les copies des candidats sont notées conjointement par les encadrants de leur pays, pour des raisons de langue et de culture, et par des coordinateurs s'assurant de la neutralité de cette correction.

Des médailles (or, argent, bronze) et des mentions honorables sont attribuées à la plupart des candidats. Il y a également des prix spéciaux : meilleure épreuve théorique, meilleure épreuve expérimentale, solution la plus originale, meilleure fille, meilleur élève membre d'un pays nouveau aux Olympiades, etc.

\section{Les deux objectifs de la préparation française}

1 Sélectionner et entraîner les candidats qui représenteront la France. Trois cents élèves (un nombre qui ne cesse de croître), répartis dans toute la France, sont préparés par leurs professeurs à passer des tests écrits (rédigés par un comité scientifique de haute volée) pour présélectionner fin mars vingt-quatre élèves de terminale ou de première année de classes préparatoires aux grandes écoles (CPGE). Les tests sont en partie différents selon le niveau. Ces présélectionnés passent une semaine intensive de travaux pratiques début mai, grâce au soutien des ENS de Cachan et de Paris, puis sont soumis à des épreuves expérimentales à l'issue desquelles cinq heureux lauréats émergent. Leur entraînement se poursuit alors par deux mois de devoirs hebdomadaires couvrant tout le programme des $\mathrm{IPhO}$ (qui est d'un niveau plutôt bac+2) corrigés à distance, suivis de deux jours à l'ENS Cachan, juste avant le départ pour Zurich.

2• Montrer au plus grand nombre possible de lycéens comment les sciences peuvent être abordées autrement qu'au travers du seul cadre du programme scolaire, leur donner un avant-goût de ce que sont les études scientifiques dans l'enseignement supérieur. Cela permet de susciter des vocations, contribue au rayonnement de la culture scientifique en France et s'inscrit tout à fait dans la mission de "Sciences à l'École ", qui est le cadre juridique et administratif des IPhO françaises.

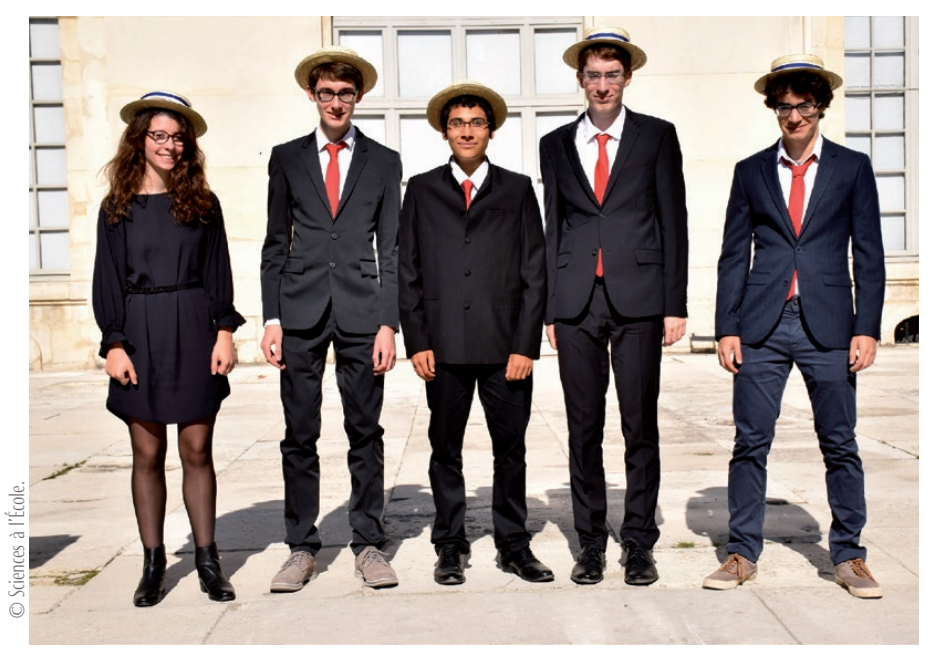

Les cinq lauréats français des IPh0 2015.

De gauche à droite : Marine Schimel (médaille d'argent), Paul Robin (médaille d’argent), Moïse Blanchard (médaille de bronze), Hughes Déprés (médaille de bronze) et Pierre Côte de Soux (médaille de bronze).

\section{Les IPhO 2015 à Mumbai}

Les résultats de la participation française aux $46^{\mathrm{es}} \mathrm{IPhO}$, qui se sont déroulées à Mumbai (Bombay, Inde) du 5 au 12 juillet 2015, sont très encourageants. Notre délégation était composée de Dominique Obert, inspecteur général de l'Éducation nationale, Isabelle Daumont et Nicolas Schlosser, enseignants, et des cinq élèves sélectionnés (quatre élèves de CPGE à Paris et un lycéen de terminale $\mathrm{S}$ en province).

L'élève de terminale (Hughes Déprés) a obtenu une médaille de bronze avec une prestation remarquable sur les épreuves expérimentales ; les étudiants de CPGE ont obtenu deux médailles de bronze et deux d'argent. Ainsi, l'ensemble de la sélection a été primé. Un prix spécial a été décerné à Marine Schimel : le prix de la meilleure candidate européenne (elle était deuxième femme au niveau mondial).

Les épreuves se sont déroulées sur deux jours. 2015 étant l'année de la lumière, elles faisaient la part belle à l'optique. Les trois épreuves théoriques portaient sur les particules solaires et les neutrinos, le principe variationnel en physique, et la conception d'un réacteur nucléaire. La partie pratique portait sur la structure hélicoïdale de l'ADN et sur les ondes de tension superficielle, étudiées par diffraction de la lumière (laser).

Durant la semaine, entre les épreuves, des visites culturelles ont été organisées pour les candidats et, dans une moindre mesure, pour les autres membres de la délégation. L'objectif de ces visites visait essentiellement la promotion de l'industrie du pays hôte. I

Bruno Jeauffroy (bruno.jeauffroy@education.gouv.fr) Président du Comité français des IPh0

Remerciements

Cette formidable opération d'ouverture culturelle et de promotion de la science serait impossible sans l'aide efficace de l'équipe de "Sciences à l'école ": ses présidents d'honneur, Claude Cohen-Tannoudji et Jean-Yves Daniel, son président Pierre Encrenaz, ses vice-présidents Pierre Desbiolles et Anne Szymzack, sa secrétaire générale Claudine Lajus et ses professeurs ressource comme Claire Bonnoit Chevalier, David Jung et Mathilde Routier. Merci et félicitations à tous, organisateurs, professeurs et élèves. 